\title{
Investigation of weight-loss expectations and weight control in obesity
}

\author{
A. McConnon, M. Raats and R. Shepherd \\ Food, Consumer Behaviour and Health Research Centre, Department of Psychology, University of Surrey, \\ Guildford GU2 7XH, Surrey, UK
}

The aim of the present abstract is to report on obese individuals' weight-loss goals and factors influencing these goals. Current guidelines recommend a target weight loss of $5-10 \%$ of original weight for successful weight control ${ }^{(1)}$. However, research has shown that this level is a great underestimation of what obese individuals consider as successful or acceptable weight loss ${ }^{(2)}$. Unmet goals or expectations in weight control can lead to negative behaviours and psychological profiles, and ultimately abandonment of weight-control efforts.

Data reported here were collected as part of the EU 6th Framework project DiOGenes, a dietary intervention trial investigating the effectiveness of high- and low-GI and -protein diets on weight maintenance, following a rapid weight-loss period, in an obese cohort. Participants were asked to indicate their target weight in kilograms in a questionnaire completed at the screening phase. A target weightloss score was then calculated by subtracting self-reported target weight from baseline weight.

Target weight-loss scores ranged from $+11 \mathrm{~kg}$ to $-88.6 \mathrm{~kg}$ and were highly correlated with baseline weight $(r-0.73, P<0.001)$, weight loss during the rapid weight-loss period $(r 0.40, P<0.001)$ and during the weight-maintenance phase of the study $(r 0.15, P=0.001)$. On average these target weights corresponded to a $25 \%$ weight loss or a mean weight loss of $25 \mathrm{~kg}$, with only $3 \%$ of the sample setting a target weight loss of $\leq 10 \%$. Weight loss necessary to reach the target weight was largely in excess of actual weight change during the rapid weight-loss period $(-11 \mathrm{~kg} v .-25 \mathrm{~kg} ; P<0.001)$ and overall weight change accounting for initial weight loss $(0.05 \mathrm{~kg} v .-25 \mathrm{~kg}$; $P<0.001)$. Differences in target weight-loss scores for gender and age were shown to be significant. Women had a significantly higher score than men $(P<0.01)$ and age was shown to be highly correlated $(r 0.11, P=0.01)$ with target weight-loss score. Regression analysis investigated the influence of gender, age, baseline weight, weight at age 20 years and weight at age 30 years on target weight-loss score. All variables were shown to significantly predict target weight-loss score, with the model explaining $72 \%$ variance $(P<0.001)$.

Target weight-loss scores were significantly negatively associated with baseline weight and positively associated with weight change, indicating that individuals with greater weight loss had higher target weight-loss scores. Women and older participants had higher weightloss expectations and target weight-loss scores were significantly predicted by previous weight history and baseline weight. Overall, this analysis reveals high weight-loss expectations in this cohort and after the intervention period target weight-loss scores were not achieved. Encouraging acceptance of realistic target weight losses in obesity management may lead to more successful treatment of obesity.

1. National Institute for Health \& Clinical Excellence (2006). Obesity: the prevention, identification, assessment and management of overweight and obesity in adults and children. NICE clinical guideline 43.

2. Elfhag K \& Rossner S (2005) Obesity Rev 6, 67-85. 